\title{
An experimental and numerical study of the vortices generated by hydrofoils
}

\author{
J. A. Szantyr, Prof. \\ R. Biernacki, Ph. D. \\ P. Flaszyński, Ph. D. \\ Gdansk University of Technology \\ P. Dymarski, Ph. D. \\ M. Kraskowski, M. Sc. \\ Ship Design and Research Centre CTO SA
}

\begin{abstract}
The article presents the results of the research project concerning the process of formation of the tip vortices shed from hydrofoils of different geometry in different flow conditions. Three hydrofoils resembling the contemporary marine propeller blades have been selected for the study. The experimental part of the project consisted of the LDA measurements of the velocity field in three cross-sections of the vortex generated by the hydrofoils in the cavitation tunnel. The numerical part of the project consisted of calculations of the corresponding velocity field by means of three computer codes and several selected turbulence models. The comparative analysis of the experimental and numerical results, leading to the assessment of the accuracy of the numerical methods, is included.
\end{abstract}

Keywords: marine propulsors; vortex generation; numerical methods; experimental techniques

\section{INTRODUCTION}

One of the most important problems in the advanced design of marine propulsors is the possibly accurate determination of the concentrated vortex structures generated by the lifting foils forming the propulsor. These vortex structures usually lead to formation of the cavitating kernels, which are responsible for the cavitation erosion of the propulsor and the rudder, for the generation of pressure pulses leading to vibration of the ship structure and for the emission of intensive hydro-acoustic signals. Development of the methods for the accurate prediction of the above described phenomena at the design stage would lead to the complete elimination of their negative consequences or at least to limiting them to the acceptable levels.

The rapidly developing methods of the Computational Fluid Mechanics are generally regarded as unable to predict accurately the intensity of the concentrated vortex structures generated by lifting foils, hence they are also unable to predict correctly the conditions for cavitation inception in these vortex structures. It is believed that the structures of the discrete representation of the fluid domain typically employed in these methods are unsuited for the cases with high velocity secondary transverse flows and that the standard turbulence models are developed and calibrated mostly for typical flat plate boundary layers, differing significantly from the vortex dominated flows. The objective of the research project presented in this paper is to develop the rules for generation of the discrete meshes especially suited for vortex dominated flows and to select the turbulence models especially effective in such flows.
This objective is achieved by the combined experimental and numerical research. Three variants of the hydrofoil were selected for the project. Their outline and section profiles resemble those of the typical contemporary marine propeller blades. The radial distribution of pitch typical for a propeller blade was reduced to zero, thus producing the foil with standard distribution of the hydrodynamic loading along the span, denoted $\mathrm{N}$ for neutral. As the distribution of the hydrodynamic loading along the span of the foil may influence significantly the process of formation of the tip vortices, two more foils were designed: one with positive linear twist along the span, denoted L for loaded, and another with negative twist along the span, denoted $U$ for unloaded. The absolute value of the twist angle in both cases was equal to 2.5 degrees at the tip, corresponding to the typical values for marine propeller blades. The details of the experimental and numerical parts of the project are described below, with the following comparative analysis of the experimental and numerical results.

\section{EXPERIMENTAL MEASUREMENTS OF THE VELOCITY FIELD AROUND THE CONCENTRATED VORTICES}

The comprehensive descriptions of the experiments with three hydrofoil models are presented in $[1,2,3]$. All three hydrofoil models were manufactured in aluminum alloy using the numerically controlled milling machine, which ensured high accuracy (Fig. 1). The span of the hydrofoil models was selected equal to $400 \mathrm{~mm}$, which located the trailing tip 
vortex approximately in the centre of the measuring section of the cavitation tunnel of CTO S.A. The hydrofoils were installed in the measuring section of the cavitation tunnel. Using the dynamometer, which enabled both measurements of the lift and drag forces and the accurate setting of the angle of attack (Fig. 2). The nominal velocity of flow in the tunnel was set to $4.0[\mathrm{~m} / \mathrm{s}]$, which ensured the turbulent flow over the hydrofoil.

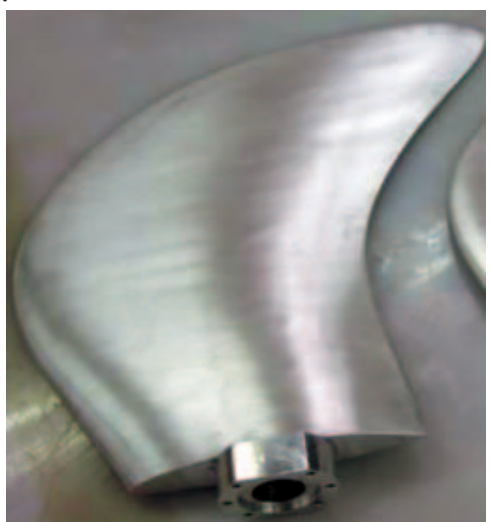

Fig. 1. The model of the hydrofoil $N$ manufactured in aluminum alloy using the numerically controlled milling machine

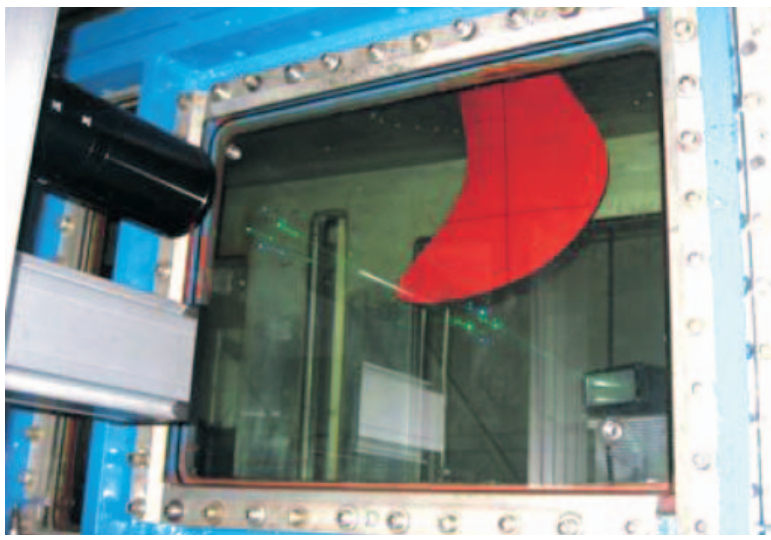

Fig. 2. The model of the hydrofoil fixed in the measuring section of the cavitation tunnel at CTO S. A.

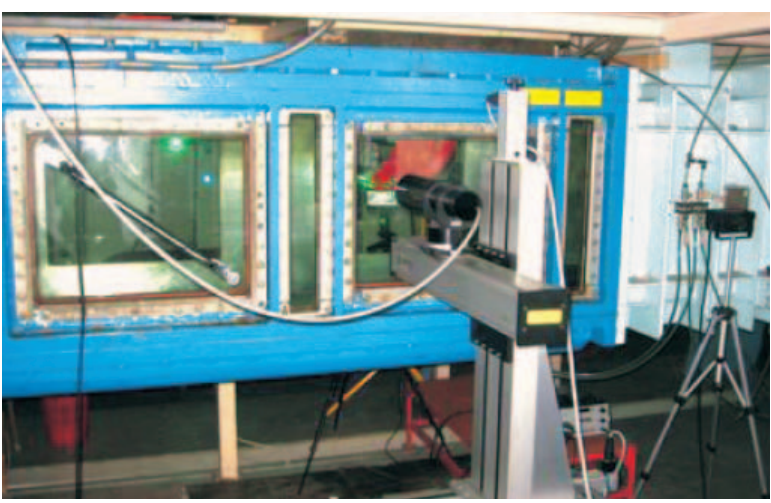

Fig. 3. The laser Doppler anemometer (LDA) ready for measurements of the velocity field around the vortex behind the hydrofoil

Each of the three hydrofoils was in turn set at three angles of attack: $+2.5 ; 0.0$ and -2.5 degrees. These values correspond to the typical range of variation of the angle of attack encountered on marine propellers. The measurements of the velocity field behind the hydrofoils were conducted by the laser Doppler anemometer (LDA - Fig. 3). The LDA system was capable of measuring two velocity component simultaneously: the axial component $\mathrm{u}$ and the transverse (vertical) component $\mathrm{v}$. The measurements were conducted in three surfaces perpendicular to the vortex axis, located at $10 \mathrm{~mm}, 70 \mathrm{~mm}$ and $300 \mathrm{~mm}$ behind the hydrofoil (Fig. 5). Apart from the velocity field, the variation of the lift and drag forces on the hydrofoils as the functions of the angle of attack were also measured.

\section{NUMERICAL PREDICTION OF THE VELOCITY FIELD AROUND THE CONCENTRATED VORTICES}

The comprehensive details of the numerical prediction of the velocity field in the vortex wake behind the hydrofoils are described in $[1,2,4,5]$. Three numerical codes were used: the commercial programs Fluent and Comet and an indigenous program Solaga developed at CTO S.A. All three programs are based on the Reynolds Averaged Navier Stokes Equations, converted into algebraic equations by means of the Finite Volume Method. The computational domain had the same cross-section as the cavitation tunnel, i.e. $800 \times 800 \mathrm{~mm}$ and it extended $1500 \mathrm{~mm}$ in front of the hydrofoil axis and $3000 \mathrm{~mm}$ behind the hydrofoil axis. This domain was divided into the block-structured grid having approximately 1.250.000 finite volumes - see Fig. 1. The size of the smallest finite volumes close to the hydrofoil surface was approximately $0.02 \mathrm{~mm}$. The following turbulence models were used in calculations:

- In the program Fluent 6.3, using the scheme MUSCL (Monotone Upstream-Centered Schemes for Conservation Laws) for the convection terms of the transport equations:

- Spalart-Allmaras

- k-epsilon RNG

- k-omega SST

- Reynolds Stress Model (RSM)

- In the program Comet:

- k-epsilon

- k-omega

- k-omega SST

- In the program Solaga - Spalart-Allmaras

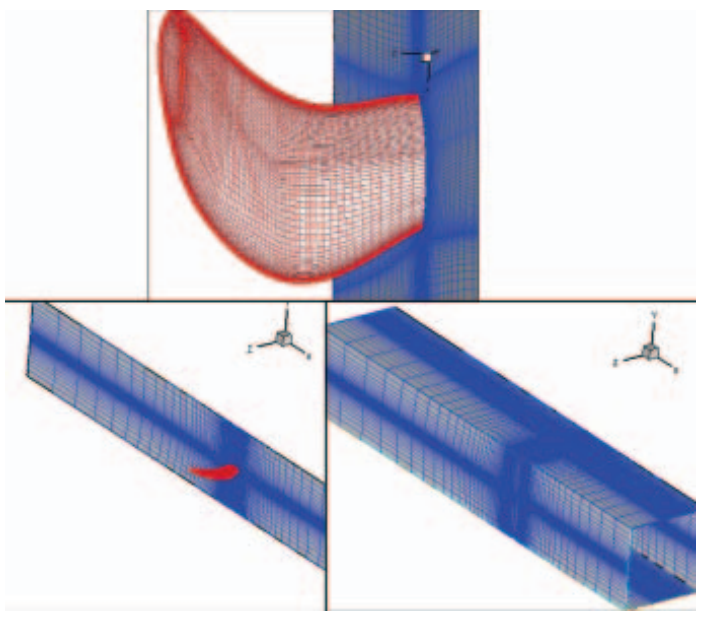

Fig. 4. The discrete mesh for numerical calculations on the hydrofoils surface (top) and on the external surfaces of the flow domain (bottom)

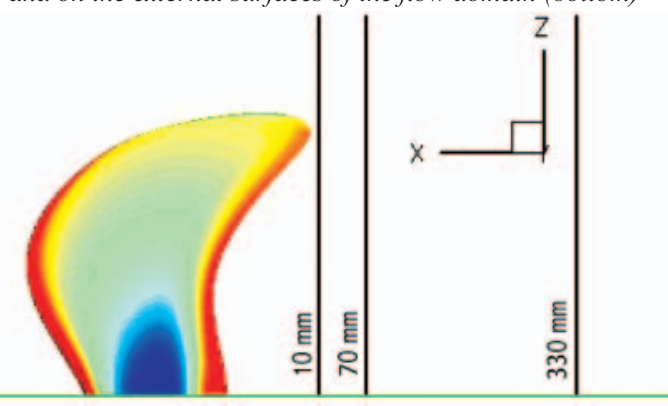

Fig. 5. The location of the three control surfaces both for measurements and calculations of the velocity field behind the hydrofoil 
The calculations reproduced the experimental conditions as closely as possible and they concentrated on determination of the axial and transverse (vertical) velocity components in the three cross-section of the vortex wake, respectively $10 \mathrm{~mm}$, $70 \mathrm{~mm}$ and $300 \mathrm{~mm}$ behind the hydrofoil trailing edge, as shown in Fig. 5.

\section{COMPARISON OF THE EXPERIMENTAL AND NUMERICAL RESULTS}

The results selected for comparison refer to two crosssections of the vortex wake, namely $10 \mathrm{~mm}$ and $70 \mathrm{~mm}$ behind the hydrofoil. At both sections, the results are presented for

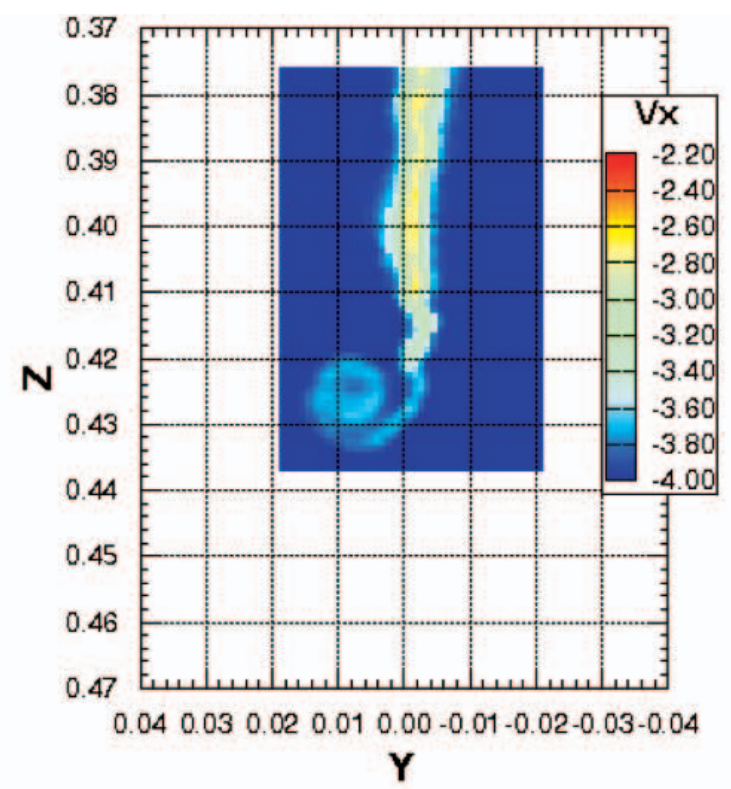

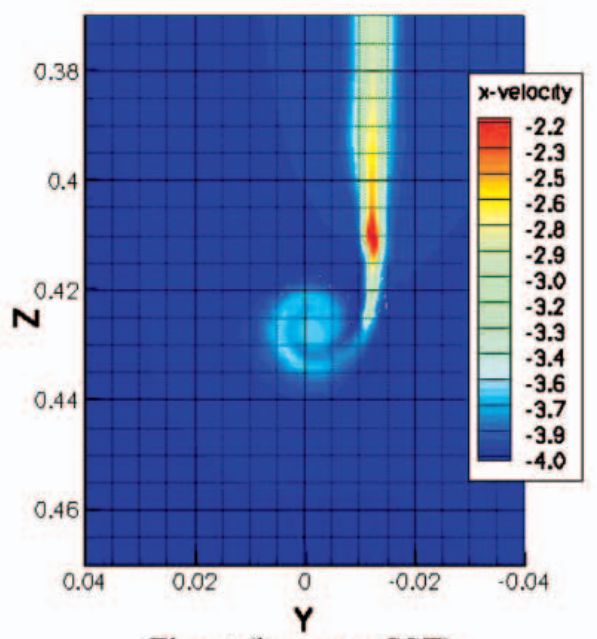

Fluent (k-omega SST)

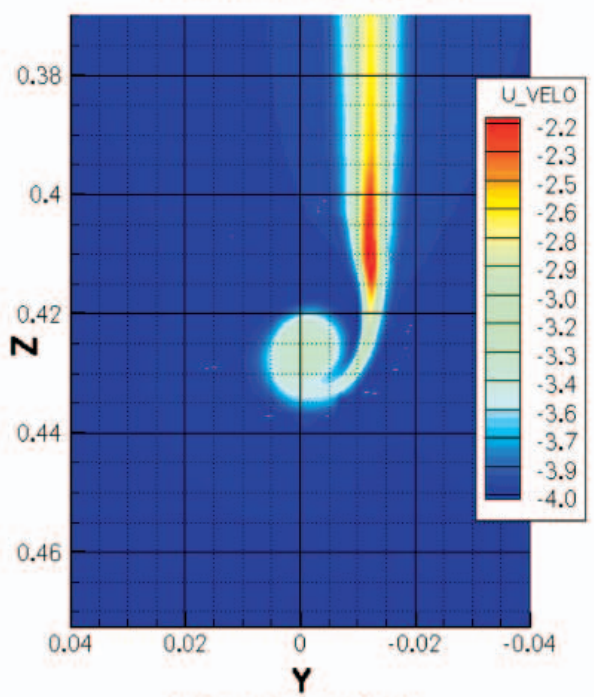

Comet (k-epsilon)

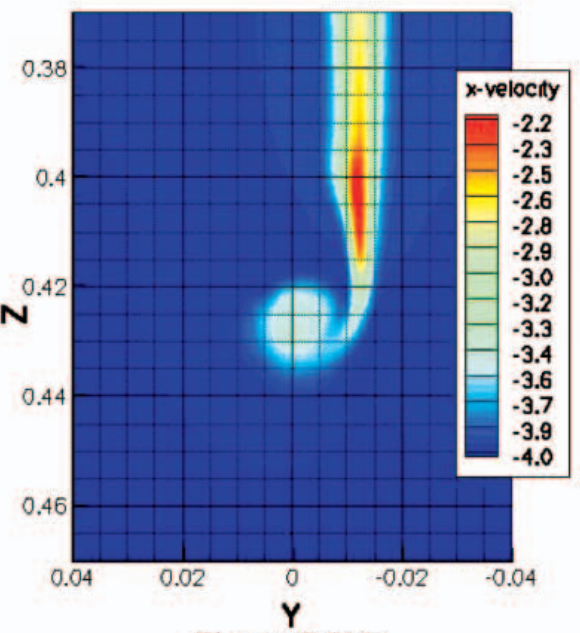

Fluent (RSM)

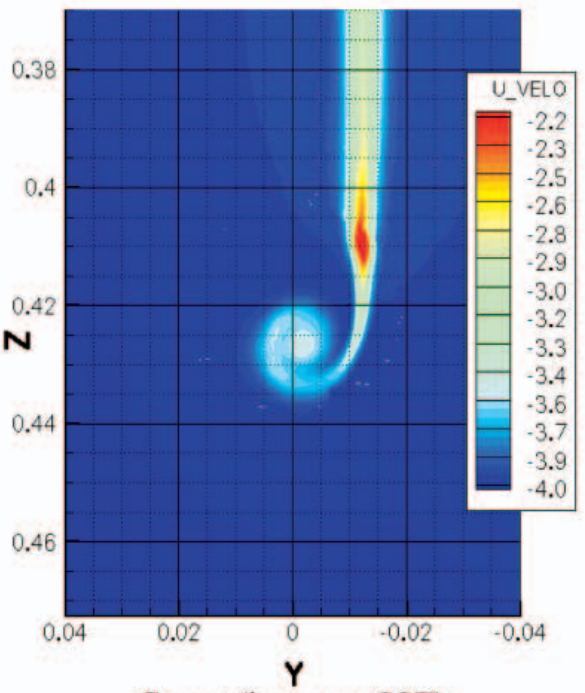

Comet (k-omega SST)

Fig. 6. The comparison of the measured (top) and calculated (below) axial component of the velocity at the control surface $10 \mathrm{~mm}$ behind the hydrofoil $L$ at the angle of attack equal +2.5 degrees 
these turbulence models which demonstrated the best ability to reproduce the experimental results. All presented results were obtained for the hydrofoil L set at the angle of attack equal to +2.5 degrees, i.e. for the case of the highest hydrodynamic loading at the tip of the hydrofoil. Consequently, the most intensive tip vortex was expected in this condition. Fig. 6 shows the measured and calculated axial velocity component at the cross-section nearest to the hydrofoil trailing edge. This velocity component is the reflection of the viscous wake behind the hydrofoil, generated in the boundary layer on its surface. The analysis of the velocity distributions shown in this figure leads to the following conclusions:

- calculations using all programs and turbulence models give the qualitatively correct picture of the viscous wake behind

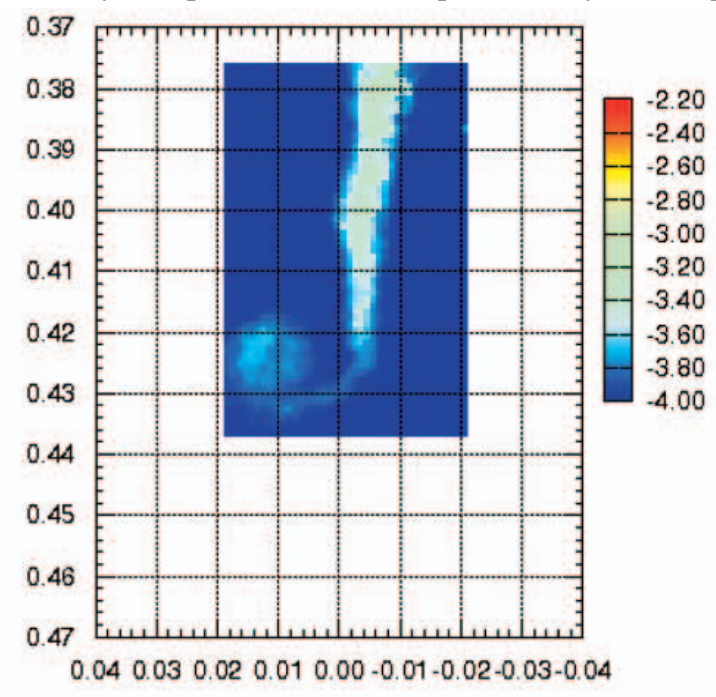

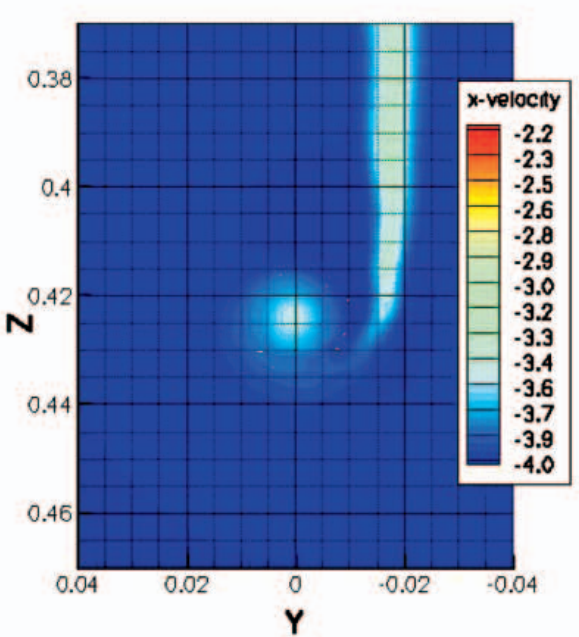

Fluent (k-omega SST)

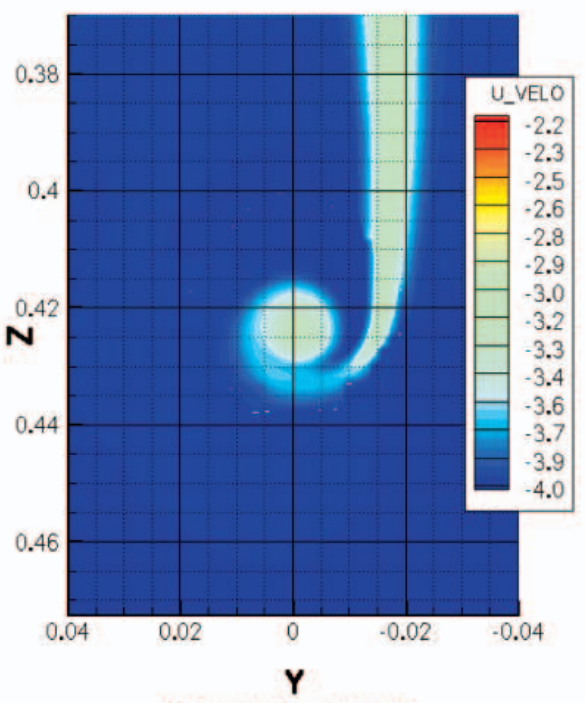

Comet (k-epsilon)

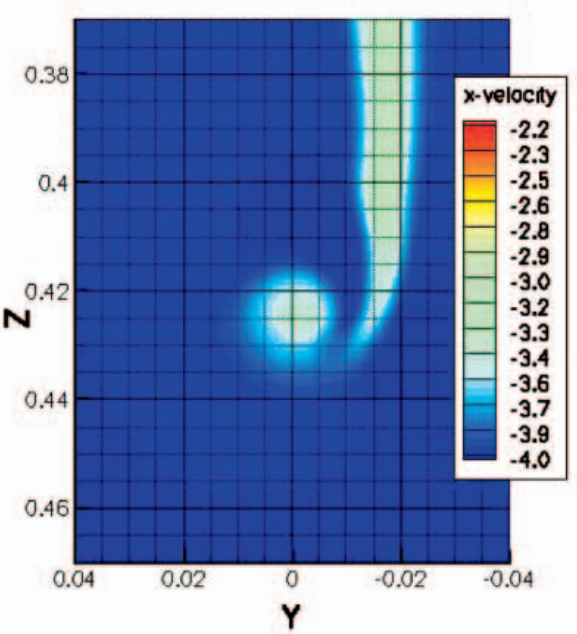

Fluent (RSM)

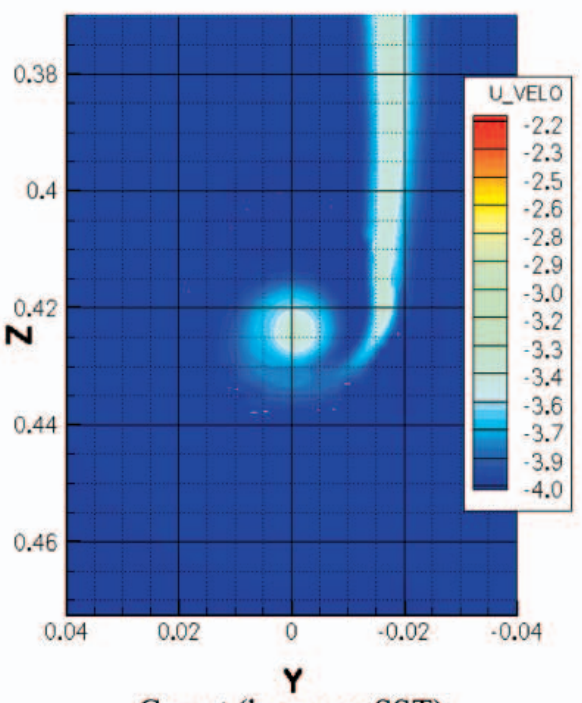

Comet (k-omega SST)

Fig. 7. The comparison of the measured (top) and calculated (below) axial component of the velocity at the control surface $70 \mathrm{~mm}$ behind the hydrofoil $L$ at the angle of attack equal to +2.5 degrees 
the hydrofoil, which agrees quite well with the results of the measurements,

- the calculated boundaries of the viscous wake are more sharply defined than those determined experimentally,

- the calculated minimum velocity in the viscous wake is markedly smaller than that detected in the course of experiments,
- this over-prediction of the minimum velocity in the wake is much stronger for the RSM and k-epsilon turbulence models than for the k-omega SST turbulence model,

- it is interesting to notice that both Fluent and Comet have produced very similar results using the same turbulence model k-omega SST.

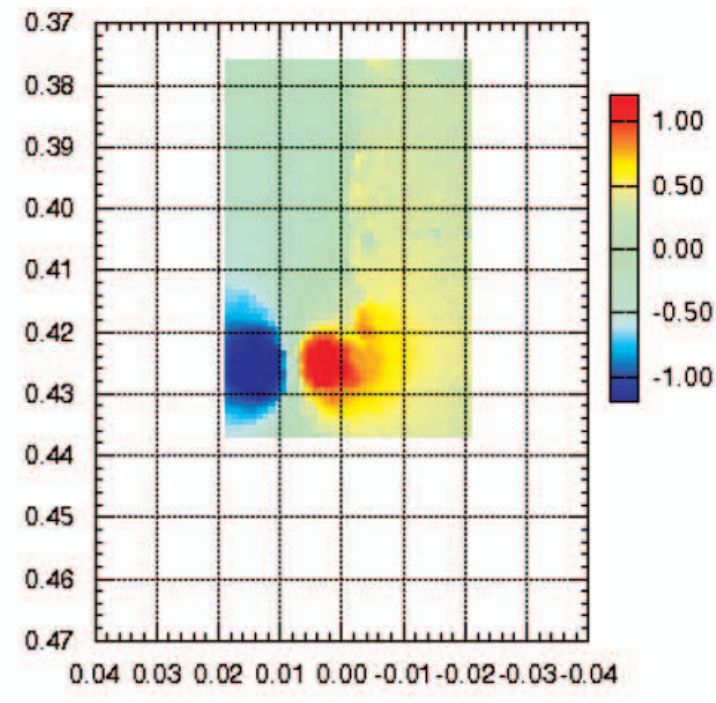

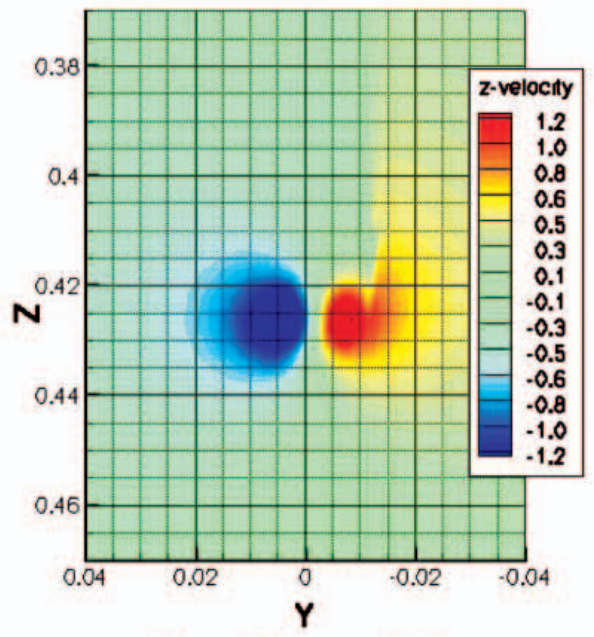

Fluent (k-omega SST)

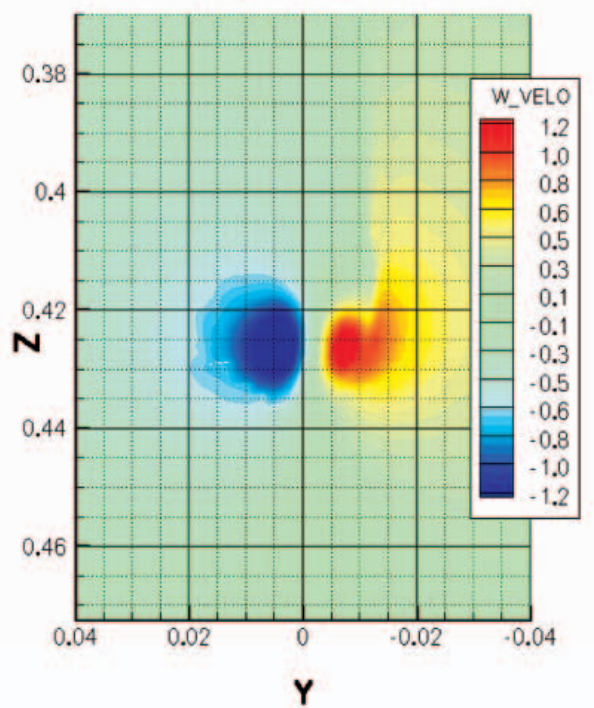

Comet (k-epsilon)

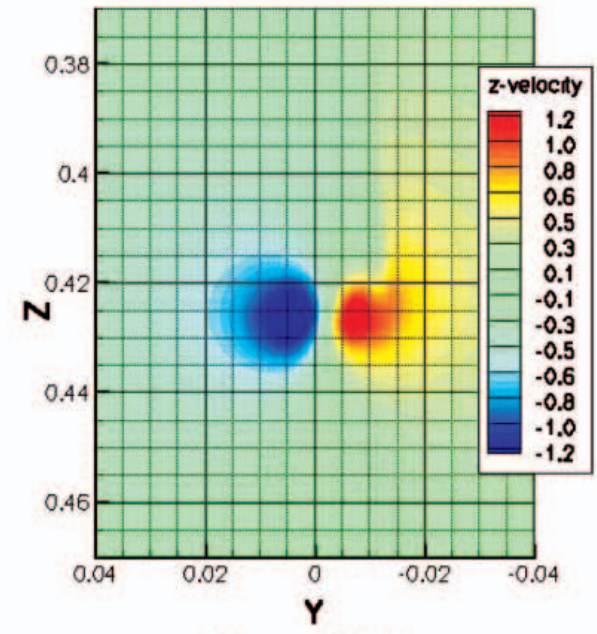

Fluent (RSM)

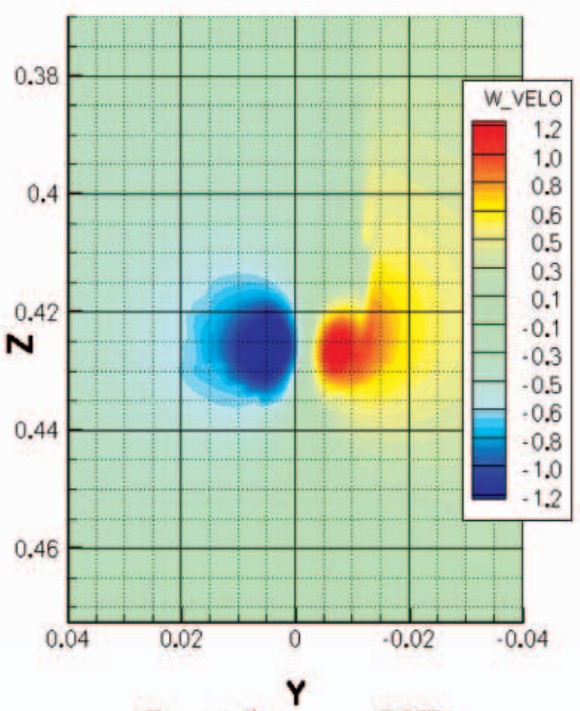

Comet (k-omega SST)

Fig. 8. The comparison of the measured (top) and calculated (below) transverse component of the velocity at the control surface $10 \mathrm{~mm}$ behind the hydrofoil $L$ at the angle of attack equal to +2.5 degrees 
Fig. 7 shows the analogical comparison of the measured and calculated axial component of the velocity at the crosssection located at $70 \mathrm{~mm}$ behind the hydrofoil trailing edge. As could be expected, at this location the viscous wake behind the hydrofoil was already significantly dispersed and damped due to the action of turbulence and fluid viscosity. This effect was correctly qualitatively reproduced in all calculations. However, again the k-omega SST turbulence model gave the best agreement with the results of experimental measurements, both in terms of the spatial distribution of the wake and the minimum velocity. Similarly as for the first cross-section, the k-epsilon and RSM turbulence models again over-predicted the minimum velocity in the wake.

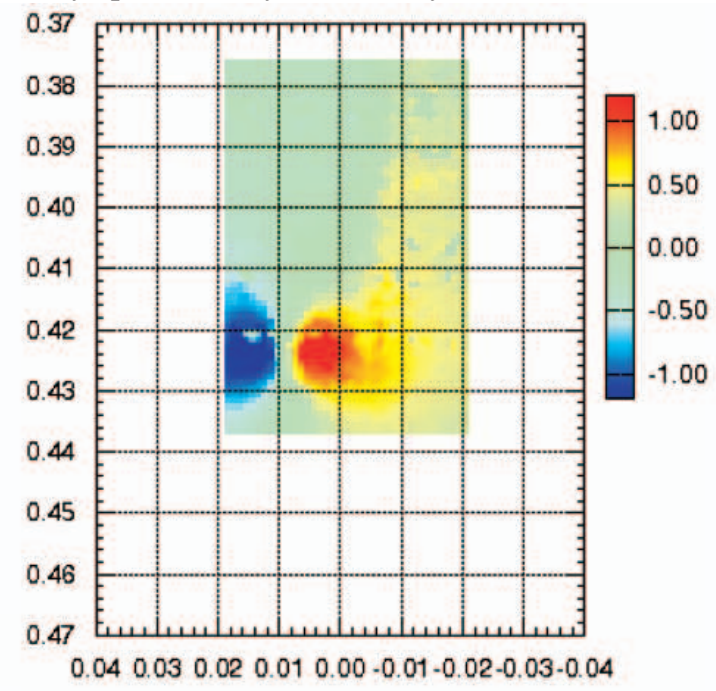

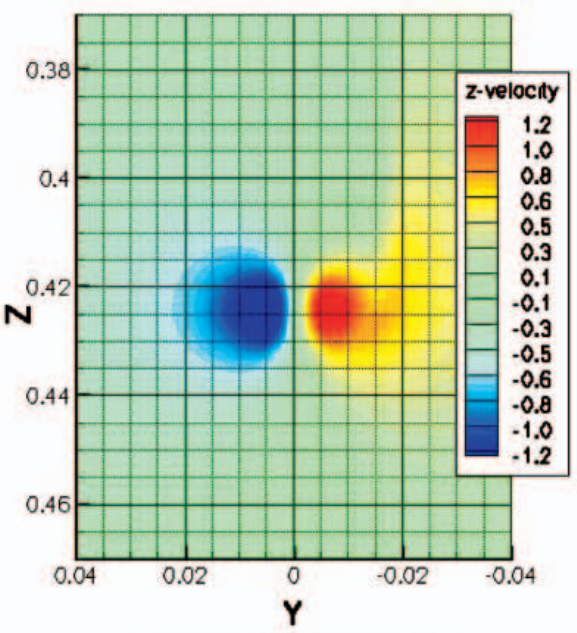

Fluent (k-omega SST)

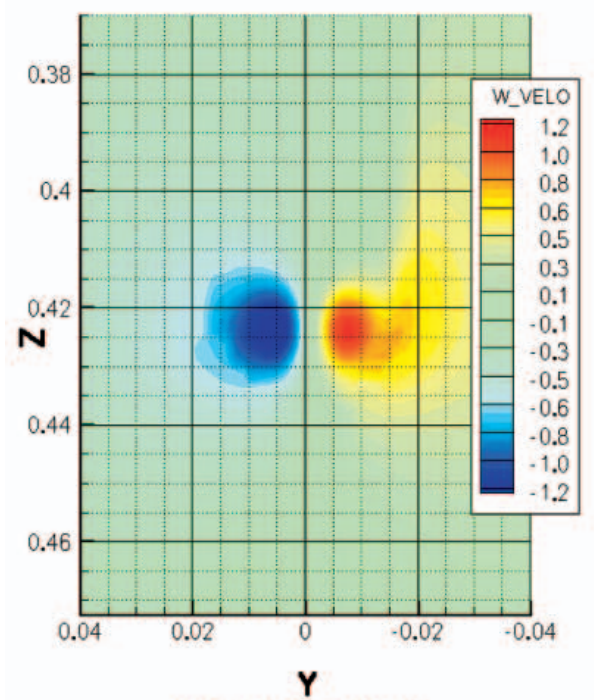

Comet (k-epsilon)

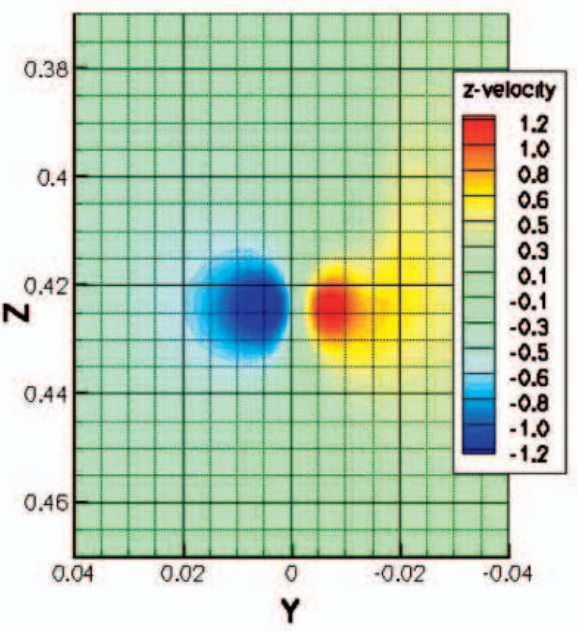

Fluent (RSM)

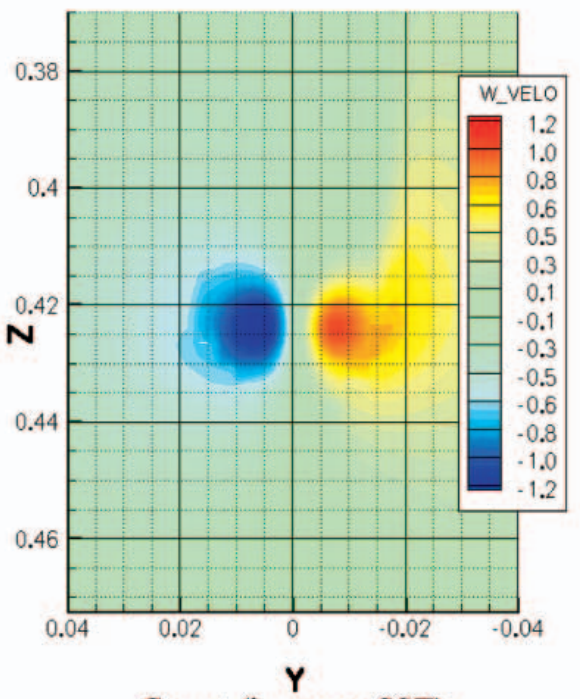

Comet (k-omega SST)

Fig. 9. The comparison of the measured (top) and calculated (below) transverse component of the velocity at the control surface $70 \mathrm{~mm}$ behind the hydrofoil $L$ at the angle of attack equal to +2.5 degrees 
In Fig. 8 the comparison of the calculated and measured transverse (vertical) component of the velocity in the wake behind the hydrofoil is presented for the cross-section located $10 \mathrm{~mm}$ behind the hydrofoil trailing edge. This velocity component may be regarded as the measure of the intensity of the tip vortex. The analysis of the velocity distributions leads to the following conclusions:

- calculations using all programs and turbulence models give the qualitatively correct picture of the intensity of the tip vortex, which agrees quite well with the results of the measurements,

- even the small details of the spatial distribution of the vertical velocity component are very well reproduced in all calculations (cf. the shape of the red field in the pictures),

- again the k-omega SST turbulence model seems to give the results closest to those obtained experimentally.

Fig. 9 presents the distribution of the horizontal component of the velocity at the cross-section $70 \mathrm{~mm}$ behind the hydrofoil. Contrary to the picture shown in Figs. 6 and 7, now there is almost no turbulent and viscous dispersion effect of the vortex visible between Figs. 8 and 9 .

Simply the vorticity is still being convected towards the centre of the tip vortex. Consequently, the distributions shown in Figs. 8 and 9 are very similar to each other. This phenomenon, detected experimentally, is confirmed by all calculations. In this case no significant differences between the respective turbulence models may be noticed.

\section{CONCLUSIONS}

After a thorough analysis of the presented results the following general conclusions may be drawn:

- the state of the art Computational Fluid Dynamics methods are fully capable of predicting the existence, location, spatial extent and intensity of the tip vortices generated by hydrofoils,

- moreover, they are also capable of predicting correctly the intensity and consequences of two mutually counteracting processes of dissipation and concentration of vorticity along the vortex,

- there are visible differences between the performance of different turbulence models and selection of the appropriate model is an important matter - in case of vortex dominated flows the k-omega SST model seems to the most promising,
- there is definitely a room for improvement of the accuracy of numerical prediction of the tip vortex flows through modification of the discrete grid structure in the flow domain, especially in the direct vicinity of the vortex.

The further stages of the research project, concerning the improvement of the performance of the CFD methods in prediction of the vortex dominated flows will be the subject of a separate article.

\section{BIBLIOGRAPHY}

1. Dymarski P., Szantyr J., Flaszyński P., Kraskowski M., Biernacki R.: Modelling of Tip Vortex Behind a Blade Using Different Turbulence Models and Different RANSE Solvers. Comparison with LDA Measurements, Proc. of the 11th Numerical Towing Tank Symposium, Brest, France, September 8-10 2008

2. Flaszyński P., Szantyr J., Dymarski P., Krasowski M.: Numerical Prediction of Vortex Generated by a Hydrofoil, Proc. of the International Symposium on Marine Propulsors, Trondheim, Norway, June 22-24, 2009

3. CTO S.A. Report No. RH-2008/T-133 Results of measurements of the velocity field in the vortex path behind the hydrofoils (in Polish)

4. CTO S.A. Report No. RH-2008/T-019 Calculations for the first variant of the discrete mesh - program Fluent (in Polish)

5. CTO S.A. Report No. RH-2008/T-020 Calculations for the first variant of the discrete mesh - programs Comet and Solaga (in Polish)

\section{CONTACT WITH THE AUTHORS}

J. A. Szantyr Prof. e-mail: jas@pg.gda.pl R. Biernacki Ph. D., e-mail: rbiernac@pg.gda.pl

P. Flaszyński Ph. D. e-mail: pflaszyn@pg.gda.pl

Faculty of Mechanical Engineering Gdansk University of Technology Narutowicza 11/12 80-231 Gdańsk

P. Dymarski Ph. D., e-mail: padym@cto.gda.pl M. Kraskowski M. Sc, e-mail: Marek.Kraskowski@cto.gda.pl

Ship Design and Research Centre CTO S.A. Wały Piastowskie 1 80-958 Gdańsk 